\title{
Anabases
}

ANABASES Traditions et réceptions de l'Antiquité

$30 \mid 2019$

Varia

\section{Le projet EPIDI : Epítetos divinos. Experiencia religiosa $y$ relaciones de poder en Hispania}

Jaime Alvar

\section{OpenEdition}

1 Journals

Édition électronique

URL : https://journals.openedition.org/anabases/10070

DOI : 10.4000/anabases. 10070

ISSN : 2256-9421

Éditeur

E.R.A.S.M.E.

Édition imprimée

Date de publication : 21 octobre 2019

Pagination : 198-202

ISSN : 1774-4296

\section{Référence électronique}

Jaime Alvar, «Le projet EPIDI : Epitetos divinos. Experiencia religiosa y relaciones de poder en Hispania »,

Anabases [En ligne], 30 | 2019, mis en ligne le 21 octobre 2021, consulté le 08 novembre 2021. URL :

http://journals.openedition.org/anabases/10070 ; DOI : https://doi.org/10.4000/anabases.10070

(c) Anabases 

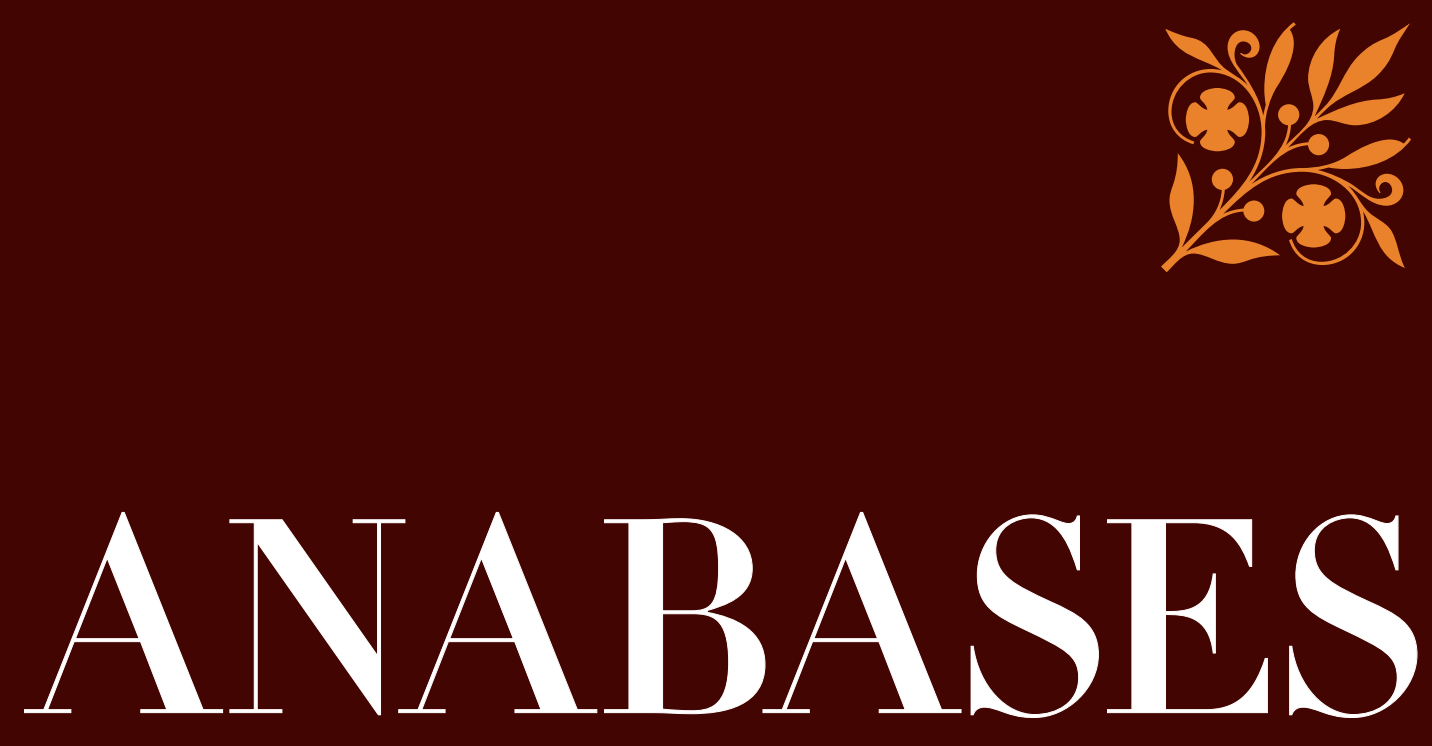

Traditions et Réceptions de l'Antiquité

\section{$\mathrm{N} \times 30$ \\ 2019}

Le sanctuaire de Gournay-sur-Aronde Le pindarisme et l'archéologie musicale Hélène en Égypte Perpétuer Ovide aux $X I V^{e}-X V I I I^{e}$ siècles II classico si fa pop Relire Marcel Detienne Freud à Pompéi 
ANABASES

Traditions et Réceptions de l'Antiquité

Revue de l'équipe de recherche E.R.A.S.M.E.

Université Toulouse-Jean Jaurès (UT2J)

Anabases dispose d'un Comité de lecture international. Chaque article envoyé à la rédaction est soumis, une fois anonymisé, à l'expertise de deux spécialistes qui rendent un rapport écrit. Les deux rapports anonymisés sont transmis à l'auteur qui tient compte des observations en vue de la publication.

\section{Comité SCIENTIFIQue}

Germaine Aujac (université Toulouse-Jean Jaurès : histoire de la géographie et des sciences antiques)

Florence Bouchet (université Toulouse-Jean Jaurès : littérature médiévale)

Hinnerk Brunns (CNRS : histoire économique et sociale ancienne et contemporaine)

Paulo Butti de Lima (université de Bari : historiographie et réception de l'Antiquité)

Luciano CANFora (université de Bari : littérature et histoire anciennes, historiographie)

Giovanna Ceserani (Stanford University : histoire intellectuelle et historiographie de la tradition classique)

Temístocles Cezar (université de Porto Alegre : historiographie moderne)

Serafina Сuомо (University of London, Birkbeck College : histoire des mathématiques et des sciences)

Paul Demont (université de Paris Sorbonne : philologie grecque et héritage classique)

Marie-Laurence Desclos (université de Grenoble II : philosophie de l'Antiquité)

Olivier Devillers (université de Bordeaux 3 - Michel-de-Montaigne : littérature et historiographie latines)

Andrea Giardina (Istituto italiano di scienze umane : histoire du monde romain et de ses réceptions)

Ève Gran-Aymerich (AIBL : histoire de l'archéologie et des transferts culturels)

François Hartog (eHess : historiographie ancienne et moderne)

Geneviève Hoffmann (université de Picardie : histoire des mondes grecs)

Christian JACOB (CNRS/EHESS : histoire comparée et épistémologie des savoirs)

Suzanne Marchand (Louisiana State University : histoire du classicisme et de l'orientalisme)

Wilfried NIPPEL (Humboldt Universität Berlin : histoire et historiographie de l'Antiquité)

Sylvie Pitria (université de Paris I-Panthéon Sorbonne : histoire et historiographie du monde romain)

Stéphane Ratтi (université de Franche-Comté - Besançon : philologie et héritage latin)

Comité de RÉdACtion

Clément Bertau-Courbières, Corinne Bonnet, Laurent bricault, Clément Bur, Adeline Grand-Clément, Anne-Hélène Klinger-Dollé, Véronique Krings, Thibaud Lanfranchi, Pascal Payen, Grégory Reimond, Sarah Rey, Catherine Valenti, Noémie VillacèQue

ÉditeUr RESPONSABLE

Clément Bur

Éditrice ADJOINTE

Catherine VALENTI

Sites Web

http://plh.univ-tlse2.fr

Revues.org : http://anabases.revues.org

Aвonnement et vente aU numéro

Éditions De Boccard - 4, rue de Lanneau - 75005 Paris

info@deboccard.com - www.deboccard.com

Tél. : 0033/(0)143260037 - Fax : 0033/(0)143548583 




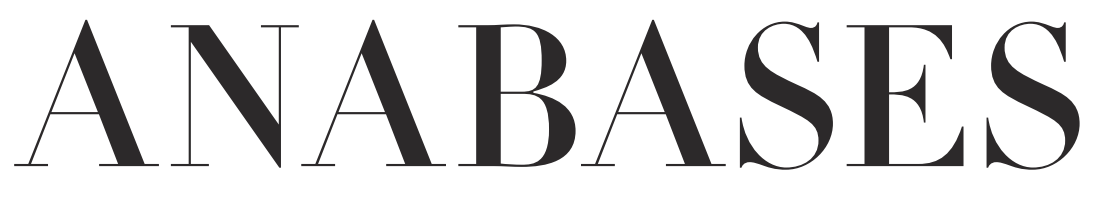

Traditions et Réceptions de l'Antiquité

$$
\begin{aligned}
& N \circ 30 \\
& 2019
\end{aligned}
$$

\section{E.R.A.S.M.E.}

Université Toulouse - Jean Jaurès 



\section{Sommaire}

ํo30 - 2019

\section{Historiographie et identités culturelles}

\section{Carole Quatrelivre}

Le sanctuaire gaulois de Gournay-sur-Aronde (Oise).

Retour sur une découverte exceptionnelle des années I970 . . . . . . . . II

\section{Traditions du patrimoine antique}

Christophe Corbier

Le pindarisme et l'archéologie musicale : style, valeur et authenticité

de la première Pythique à l'époque moderne . . . . . . . . . . . . . . .

Arnaud AmiLIEN

Hélène en Égypte : Hérodote en dialogue avec l’épopée . . . . . . . . . .

\section{Archéologie des savoirs}

Sébastien Cazalas

Au jardin des exempla. Rhétorique et stratégie de l'exemplum antique

dans l'œuvre politique de Jean Juvénal des Ursins (I388-ı473) . . . . . . 7 7

Dossier - Perpétuer Ovide : aspects moraux, éditoriaux,

linguistiques et culturels $\left(\mathrm{XIV}^{\mathrm{e}}-\mathrm{XVIII}^{\mathrm{e}} \mathrm{s}\right.$.)

Francesca Dell'Oro

Introduction $\ldots \ldots \ldots \ldots$. . . . . . . . . . . . . . 89

Hélène Casanova-Robin

L’audace châtiée : Phaéton, Actéon et Icare dans la tradition latine jusqu'à la Renaissance, tours et détours d'un symbolisme . . . . . . . . 9 93 
Dylan Bovet

Le commentaire latin des Métamorphoses d'Ovide : pratiques humanistes et évolutions de Regius-Micyllus (I543) à Burmann-Heinsius (I727) . . . . III

Martine FurNo

Ovide en classe, ou un auteur en éclats . . . . . . . . . . . . . I27

Basil NELIS

D’un Ovide chrétien à un Ovide burlesque, du Moyen Âge au Grand Siècle : continuités et changements dans la traduction et dans l'illustration des Métamorphoses perçus à travers deux éditions du xvII ${ }^{\mathrm{e}}$ siècle . . . . . . . I I43

Olivier ThÉvenAz

Épilogue ....................... I6

\section{Actualités et débats}

Tiphaine-Annabelle BEsnard

2019 : l'année pop des musées d'archéologie. Retour sur l'exposition

romaine Il classico sifa pop. Di scavi, copie e altripasticci . . . . . . . . . . I I7I

\section{Lire, relire la bibliothèque des sciences de l'Antiquité}

Jean-Pierre Albert

Le premier Detienne : une relecture de

“ La notion mythique d"A $\lambda \hat{n} \theta \varepsilon 1 \alpha$ » $\left(R E G\right.$, I96o, p. 27-35) . . . . . . . . . . $\quad{ }_{779}$

Marcel Detienne

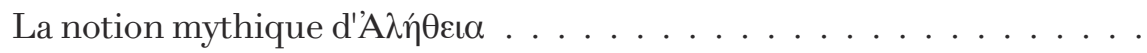

\section{Ateliers de l'histoire}

Antiquités numériques (coordonné par Elodie Guillon) ( $n^{\circ} 1$ )

Élodie GuILlon

Introduction

Jaime Alvar

Le projet EPIDI : Epítetos divinos.

Experiencia religiosa y relaciones de poder en Hispania . . . . . . . . . . . I98 Les mots de l'Antiquité (coordonné par Magali Soulatges) ( $n^{\circ} 10$ )

Jack Thомаs

L’Antiquité dans les toponymes de l'État de New York . . . . . . . . . . . 202

Actualité du théâtre (coordonné par Malika Bastin-Hammou) ( $\left.n^{\circ} 4\right)$

Mathieu FERrand

“ Avons-nous perdu le Soleil ? / Ou l'avons-nous chassé ? » 
Thyeste de Sénèque, traduit par Florence Dupont.

Mise en scène de Thomas Jolly (Avignon, 20I8) . . . . . . . . . . . . . 2 2I4

Voyages et Voyageurs (coordonné par Véronique Krings) ( $n^{\circ} 11$ )

Claude AzIzA

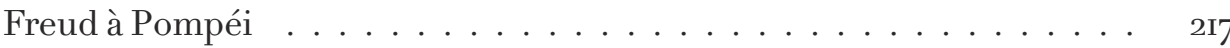

\section{Comptes rendus}

Lucile Arnoux-Farnoux et Polina Kosmadaki (dir.)

Le double voyage : Paris-Athènes (Igrg-Ig39) (Catherine Valenti) . . . . . . 225

Sandra Boehringer et Daniele Lorenzini (dir.)

Foucault, la sexualité, l'Antiquité (Jan Nelis) . . . . . . . . . . . . . . . . 226

Corinne Bonnet, Nicole Belayche, Marlène Albert Llorca,

Alexis Avdeeff, Francesco Massa, Iwo Slobodzianex (dir.)

Puissances divines à l'épreuve du comparatisme. Constructions,

variations et réseaux relationnels (Geneviève Hoffmann) . . . . . . . . . . 228

Shane ButLer (éd.)

Deep Classics, Rethinking Classical Reception (Jan Nelis) . . . . . . . . . . 23o

Zeynep ÇELIK

About Antiquities: Politics of Archaeology in the Ottoman Empire (Jorge Elices Ocón) . . . . . . . . . . . . . . . . . 231

Xavier Deru et Germaine Leman-Delerive (éd),

Franz Cumont, Comment la Belgique fut romanisée (Vivien Barrière) . . . 234

Olivier Devillers, Breno Battistin Sebastiani (éd.)

Sources et modèles des historiens anciens (Arnaud Saura-Ziegelmeyer) . . $\quad 235$

Mara Fazio, Pierre Frantz et Vincenzo De Santis (dir.)

Les Arts du spectacle et la référence antique dans le théâtre

européen (I760-I830) (Arnaud Saura-Ziegelmeyer) . . . . . . . . . . . . . . 237

Jérémy Guedu et Barbara Meazzi (dir.)

La culture fasciste entre latinité et méditerranéité (I880-1940), in Cahiers de la Méditerranée 95, (Andrea Avalli) . . . . . . . . . . . . . . . 239

Marie-Laurence HAAck (éd.), avec la collaboration de Martin MiLler, Les Étrusques au temps du fascisme et du nazisme (Jan Nelis) . . . . . . . . . 24I

Arlene Holmes-Henderson, Steven Hunt et Mai Musié (éd.)

Forward with Classics. Classical Languages in Schools and Communities

(Charlotte Tournier) . . . . . . . . . . . . . . . . . . . . . . .

Daniel Jew, Robin Osborne et Michael Scotт (éd.)

M. I. Finley. An Ancient Historian and his Impact (Hinnerk Bruhns) . . . . $\quad 244$ 
Kostas Kalimtzis,

An inquiry into the philosophical concept of scholê.

Leisure as a Political End (Florent Rouzade) . . . . . . . . . . . . . . . . . 246

Anne-Hélène KLINGER-Dollé

Le De sensu de Charles de Bovelles. Conception philosophique

des sens et figuration de la pensée. Suivi du texte latin du De sensu,

traduit et annoté (Laure Hermand-Schebat) . . . . . . . . . . . . . 248

Egidia Occhipinti

The Hellenica Oxyrhynchia and Historiography:

New Research Perspectives (Anne de Cremoux) . . . . . . . . . . . . . . . . 249

Laurent OLIVIER (dir.)

La mémoire et le temps. L'ouvre transdisciplinaire

d'Henri Hubert (I872-I927) (Sarah Rey) . . . . . . . . . . . . . . . 25I

Gabriella Pinonti et Corinne Bonnet (dir.),

Les dieux d'Homère. Polythéisme et poésie en Grèce ancienne,

Kernos, Supplément 3r. (Sandya Sistac) . . . . . . . . . . . . . . . 253

Rabun TAYLoR, Katherine W. Rinne et S. Kostof

Rome. An Urban History from Antiquity to the Present

(Cyrielle Landrea) . . . . . . . . . . . . . . . . . . . 255

Wyger Velema et Arthur Weststeisn (éd.)

Ancient Models in the Early Modern Republican Imagination

(Paulo Butti de Lima) . . . . . . . . . . . . . . . . . . . 256

Philip WaLsh (éd.)

Brill's Companion to the Reception of Aristophanes

(Malika Bastin-Hammou) . . . . . . . . . . . . . . .

Richard WARren

Art Nouveau and the Classical Tradition (Lucien Calvié) . . . . . . . . . 26o

Jesse Weiner, Benjamin Eldon Stevens et Brett M. Rogers (éd.)

Frankenstein and Its Classics. The Modern Prometheus

from Antiquity to Science Fiction (Mathieu Scapin) . . . . . . . . . . . . . 26r

Nigel G. WiLson

From Byzantium to Italy. Greek Studies in the Italian Renaissance,

deuxième édition (Luigi-Alberto Sanchi) . . . . . . . . . . . . . . 262

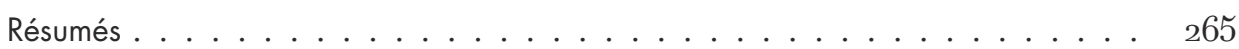

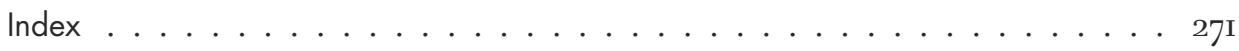




\section{L'atelier de l'histoire : \\ chantiers historiographiques}



les lacunes et les aspects disparates d'une documentation ancienne et nécessairement hétérogène; il nous oblige à une formalisation accrue des données. L'atelier laisse donc toute latitude aux auteurs pour exprimer les difficultés rencontrées et les solutions envisagées, dans l'idée d'un retour d'expérience pour leurs pairs.

Les recherches présentées ici sont menées par des spécialistes de l'Antiquité, car avec le numérique il n'est jamais question de faire table rase du passé et des connaissances de l'historien. Au contraire, il s'agit bien de mettre à profit des outils actuels et performants bases de données, logiciels de géolocalisation, de modélisation de réseaux, d'analyses statistiques complexes, etc. - dans un dialogue fructueux avec le savoir-faire et l'œil avisé du spécialiste. Face à des corpus de données croissant rapidement, qui nous brossent le tableau d'un passé incroyablement dynamique et mouvant, force est de constater que nous avons besoin d'aide pour regrouper, trier et comparer nos données. Ces outils, utilisés à propos, nous facilitent ainsi la compréhension et la restitution de la complexité des sociétés anciennes et de celle des sociétés modernes qui les lisent.

\author{
Élodie Guillon \\ Université Toulouse-Jean Jaurès \\ ERCP MAP (741182) PLH - ERASME \\ 5 Allées Antonio Machado \\ 31058 Toulouse Cedex 9 \\ elodie.guillon@univ-tlse2.fr
}

\title{
Le projet EPIDI : Epítetos divinos. \\ Experiencia religiosa y relaciones de poder en Hispania
}

Jaime Alvar

Le projet de recherche EPIDI (Epitetos divinos. Experiencia religiosa y relaciones de poder en Hispania, HAR20I7-84789-C2-2-P $)^{3}$ envisage deux buts en parallèle. Le premier est d'approfondir la connaissance des épithètes comme élément spécifique de la divinité et

3 Ce projet est financé par le ministère espagnol de « Science, Innovation et Université », avec l'aide des fonds FEDER. Il fait partie d'un projet coordonné par Clelia Martínez Maza de l'Université de Málaga, intitulé “Voies d'accès au divin ». Les membres de l'équipe de travail sont: Jaime Alvar, chercheur responsable, Valentino Gasparini, Alejandro Beltrán, José Carlos López Gómez et Beatriz Pañeda, tous appartenant à l'Universidad Carlos III de Madrid, ainsi que la boursière du projet LARNA, María Fernández Portaencasa (dirigée par Valentino Gasparini). 
comme outil de construction du divin par les acteurs du culte. Il s'agit clairement d'une voie d'accès au divin, mais en même temps, les dieux se modèlent à travers les dénominations forgées par leurs dévots. Les épithètes divines changent en vertu des circonstances sociohistoriques au moment de l'invocation et, par conséquent, redéfinissent les qualités et les potentialités des divinités, affectant directement ce qui a été appelé ces dernières années « l'agentivité divine ». Le choix de l'épiclèse oscille entre régulation normative et expérience personnelle, et se projette socialement à travers la visibilité du témoignage épigraphique. Étant donné l'ampleur du sujet, notre projet se propose d'analyser les épithètes évoquant le pouvoir et sa relation avec les formes d'organisation politique et les expressions de la dépendance.

Le second objectif est intimement lié au premier, car il s'agit d'élaborer une base de données avec tous les documents épigraphiques qui mentionnent des épithètes divines dans les trois provinces romaines d'Hispanie. C'est de la partie numérique du projet EPIDI dont il va être question $\mathrm{ici}^{4}$.

Ce n'est pas la première fois que notre équipe de recherche s'attèle à la conception d'une base de données. Le projet précédent, financé aussi par le ministère espagnol de “Science, Innovation et Université », portait sur l'étude des “cultes orientaux » en Hispanie $^{5}$. Deux monographies ont été publiées comme résultat de la recherche ${ }^{6}$; une troisième est en cours d'édition ${ }^{7}$. Le premier volume de cette trilogie a été publié en 2012 ; en 2018 a été réalisée, en outre, une édition en ligne, qui est la base de la partie numérique du projet EPIDI et qu'il convient donc de présenter.

Ce catalogue des cultes isiaques de la péninsule ibérique comporte actuellement 200 documents environ. Les documents considérés en relation avec le culte, mais qui n'y appartiennent pas directement, sont regroupés dans une rubrique "Hors Catalogue », pour éviter les erreurs. La volonté d'offrir un catalogue ouvert s'explique par deux objectifs essentiels : d'une part, permettre l'actualisation et l'enrichissement des contenus grâce aux avancées de la recherche; d'autre part, inclure les activités de recherche de tous les collègues intéressés par le sujet, qui peuvent bénéficier du financement public du projet. La base du catalogue, toujours en cours de réalisation, se présente comme une fiche, qui, dans la mesure du possible, sera accompagnée de l'image correspondante, pour offrir l'information la plus complète possible. Il est également prévu de proposer une carte interactive pour géolocaliser les documents.

4 Je laisserai donc de côté les aspects spécifiquement historiques, qui sont en rapport étroit avec le projet MAP mené par Corinne Bonnet à Toulouse et qui nous a généreusement accueillis au mois de janvier 2019. L'excellence de ce projet fait qu'il a été un modèle extraordinaire pour le projet EPIDI tant du point de vue théorique que pour ce qui concerne la méthode.

5 Projet ORINS (Oriental Religions in Roman Spain, HAR2014-52531-P).

6 J. Alvar, Los cultos egipcios en Hispania, Besançon, Presses universitaires de FrancheComté, 2012 ; J. Alvar, El culto de Mitra en Hispania, Madrid-Besançon, DykinsonPresses universitaires de Franche-Comté, 2018.

7 J. Alvar, Los cultos de Mater Magna y Atis en Hispania, Madrid-Besançon, DykinsonPresses universitaires de Franche-Comté, 2019 (sous presse). 
Pour le développement du projet nous avons reçu l'appui de la Bibliothèque des Humanités de la Universidad Carlos III de Madrid. Ses techniciens ont créé la fiche pour la base de données avec une interface web, afin de pouvoir récupérer l'information contenue dans chacune des fiches du catalogue. L'enregistrement des fiches a été effectué par les membres du groupe de travail, Beatriz Pañeda et José Carlos López. Ensuite, les techniciens ont développé la carte de géolocalisation des documents. Il est possible de naviguer de la fiche à la carte et vice versa. Le résultat du produit est hébergé sur le site web de l'université : http://www.uc3m.es/gens-isiaca. Une fois accomplie la publication on line de la gens isiaca, nous avons commencé, au mois de mai, le versement de l'information relative au culte de Mithra dans la base de données, pour créer le catalogue correspondant à ce culte sur la même plateforme. Enfin, quand la monographie correspondant aux cultes de Mater Magna et Attis sera publiée ${ }^{8}$, nous créerons le troisième catalogue.

La mise en œuvre du projet EPIDI nous conduit à préparer une nouvelle base de données, inspirée du travail précédent, avec les épithètes connues dans l'épigraphie votive de la péninsule ibérique. Dans cette perspective, nous avons élaboré un fichier Excel, avec plusieurs rubriques permettant de décrire et d'enregistrer la totalité de l'information pertinente. L'équipe a analysé un échantillon représentatif d'environ 200 documents épigraphiques, de manière à concevoir une fiche modèle satisfaisante pour accueillir les informations significatives. Les rubriques (qui correspondent à des colonnes Excel) sont les suivantes :

"Des rubriques décrivant les inscriptions : théonymes, épithètes, genre de la divinité, texte complet de l'inscription, nombre de dédicants, statut de ces derniers"

"Des rubriques de datation : terminus ante quem et post quem, critère pour la datation"

"Des rubriques de localisation: province romaine, conventus, oppidum, ager, país, communauté autonome/district, province, municipe, lieu, coordonnées"

"Des rubriques sur l'objet portant l'inscription : lieu de dépôt, numéro d'inventaire, type de support, matériel"

Les dernières rubriques sont destinées à l'information documentaire et à la bibliographie; on a destiné, donc, des colonnes spécifiques pour CIL, AE, HEp, Varia et les références bibliographiques.

Évidemment, en remplissant les colonnes avec l'information épigraphique, on rencontre des problèmes de nature diverse, qu'il faut résoudre lors de réunions périodiques.

Étant donné l'énorme variété des possibilités de classification de l'information épigraphique, il est difficile de saisir toute la richesse ou, au contraire, les faiblesses documentaires dans des cellules à listes déroulantes fermées ${ }^{9}$. En effet, il y a des inscriptions qui comportent plusieurs théonymes ou des noms personnels avec des fonctions différentes. En conséquence, l'avantage d'homogénéisation que procurent ces listes déroulantes n'est pas toujours valable, et on a dû les éviter quand l'information ne s'y adapte pas.

8 Cf. note $\mathbf{n}^{\circ} 5$.

9 En effet, pour l'harmonisation de la saisie des données, nous avons créé pour certaines rubriques des listes déroulantes à choix multiples. 
Cette question de l'homogénéisation s'est en fait posée dès que les membres du groupe de travail ont eu fini de remplir le fichier Excel avec les données regroupées par provinces. Nous avons alors constaté la nécessité de régulariser le contenu des différentes rubriques. Un exemple: nous avons constaté différentes formes d'inclusion des théonymes dans le fichier Excel. Quelques chercheurs ont opté pour l'inclusion du théonyme tel qu'il apparaît dans le texte épigraphique, d'autres ont transcrit le théonyme en espagnol ; une troisième alternative a été de l'inclure en latin, au nominatif. C'est cette dernière solution que nous avons décidé de retenir. Nous sommes donc actuellement en train d'uniformiser la restitution des théonymes, dans la forme approuvée, en respectant les lettres manquantes entre parenthèses quand il s'agit d'abréviations ou entre crochets en cas de restitution.

En revanche, il y a d'autres décisions que nous ne pouvons pas prendre en ce moment, en raison du volume de travail que cela suppose. C'est par exemple le cas de la création d'une nouvelle colonne spécifique avec la profession/charge de la personne dédicante, car jusqu'à présent cette information était incluse dans une colonne intitulée « Dédicant.e (personne) ». La tâche qui suppose l'adaptation du fichier Excel à cette décision est si lourde que nous avons préféré la remettre à une phase ultérieure.

Pour donner une idée de l'ampleur du catalogue, précisons que notre équipe a dépouillé presque 900 inscriptions votives de Lusitanie, dont 400 contiennent une épithète. En Bétique, on compte 300 inscriptions et en Tarraconèse plus de 675 avec épithète. Les provinces africaines de Cyrénaïque et Tingitaine, déjà traitées, ont fourni presque I5o documents en latin valables pour notre propos ${ }^{10}$. La deuxième phase mentionnée consistera à transférer le contenu des fichiers Excel sur la plateforme destinée à être visible sur le web. On a prévu ce transfert pour la troisième année du projet, entre janvier et mars 2020. C'est alors qu'on introduira les modifications qui sont apparues nécessaires lors de l'élaboration des fiches Excel.

Nous espérons que le catalogue des épithètes divines présentes dans le corpus épigraphique d'Hispanie permettra aux chercheurs de travailler non seulement sur ces qualificatifs, mais aussi sur les théonymes, les dédicant.e.s, les donations, les supports, ainsi que sur les aspects sociaux, chronologiques et géographiques concernés. Même si le projet EPIDI n'envisage que les épithètes de pouvoir, la base de données offrira un panorama global de l'épigraphie votive en Hispanie, dont l'utilité débordera son cadre initial. L'accès ouvert sera à disposition des chercheurs sur le site de l'Universidad Carlos III de Madrid à la fin de l'année 2020, date coïncidant avec la fin du projet.

Pour conclure, un objectif additionnel de cette base de données est d'avoir la possibilité d'établir des rapports entre une information susceptible d'être régulièrement actualisée et celle provenant d'autres régions de l'Empire ${ }^{11}$. C'est le cas des provinces

10 Le projet dirigé par Valentino Gasparini, “ Lived Ancient Religion in North Africa » (LARNA), se déroule en parallèle avec EPIDI. Nous en profitons pour établir conjointement des catégories pertinentes pour les deux projets, et élaborer des fiches adaptées, car on envisage que la base de données de LARNA soit hébergée au même endroit, sur le site de l'Universidad Carlos III de Madrid.

1 Le choix du format Excel, simple en apparence, permet en outre d'envisager des exports faciles vers d'autres bases de données qui pourraient être intéressées par notre démarche et nos données (le projet MAP, par exemple), car notre projet a vraiment pour objectif de rendre disponibles ces données. 
de l'Afrique du Nord, qui seront incluses dans notre domaine grâce au fait que le projet LARNA (“Lived Ancient Religion in North Africa », conduit par Valentino Gasparini) a son siège, tout comme le projet EPIDI, dans l'Institut d'Historiographie Julio Caro Baroja de la Universidad Carlos III de Madrid (www.uc3m.es/IHJCB).

\author{
Jaime Alvar \\ Universidad Carlos III de Madrid \\ Instituto de Historiografía \\ C/ Madrid 126 \\ 28903 Getafe (Espagne) \\ jalvar@hum.uc3m.es
}

\title{
Les mots de l'Antiquité (10)
}

\section{L'Antiquité dans les toponymes de l'État de New York'}

\section{Jack Thомаs}

L'étude des noms de lieux, la toponymie, a une longue histoire. Elle peut renvoyer à la chronologie des couches successives de peuplement dans des pays à l'histoire longue, des toponymes pouvant disparaître suite à de nouvelles vagues de peuplement. Dans des pays de création récente, comme aux États-Unis, par exemple, si certains toponymes amérindiens subsistent, beaucoup d'autres ont été remplacés par une multitude de noms de lieux inspirés des paysages, des héros nationaux ou régionaux, des pionniers et fondateurs, de la religion ou des vertus morales des colons et de leurs descendants ${ }^{2}$.

1 L'article s'appuie principalement sur deux études : Wilbur Zelinsky, "Classical Town Names in the United States, The Historical Geography of an American Idea", Geographical Review 57/4 (1967), p. 463-495 ; William R. FARrell, Classical Place Names in New York State. Origins, Histories and Meanings, illustrations de Bettina B. Chapman et Carolyn I. Coit, Jamesville, New York, Pine Grove Press, 2002.

2 Voir, par exemple, Christian Montès, “ La toponymie comme révélateur de la construction identitaire d'un empire : (re)nommer les capitales étatsuniennes », L'Espace géographique 37 (2008/2), p. 106-116. 
ANABASES

Traditions et Réceptions de l'Antiquité

Revue de l'équipe de recherche E.R.A.S.M.E.

Université Toulouse-Jean Jaurès (UT2J)

NoRMES RÉDACTIONNELLES

ANABASES publie des articles dans cinq langues : français, anglais, allemand, italien et espagnol.

Les articles ne dépasseront pas 35000 signes et seront conformes aux normes de la revue,

disponibles sur le site web : http://plh.univ-tlse2.fr

Les articles seront pourvus d'un résumé en français et en anglais, ainsi que de six à huit mots-clés dans ces deux langues.

Les articles pourront être accompagnés de planches en noir et blanc.

Les comptes rendus compteront de 4500 à 6000 signes.

Site web avec Présentation, Sommaire de tous les Numéros et Bulletin d’Abonnement

http://plh.univ-tlse2.fr

Revues.org : http://anabases.revues.org

\section{Courrier}

Pour les articles :

Clément Bur (clement.bur@univ-jfc.fr)

Catherine VALENTI (catherine.valenti@univ-tlse2.fr)

Pour les comptes rendus :

NoémieVILlacÈQue (noemie.villaceque@univ-reims.fr)

Université Toulouse-Jean Jaurès (UT2J)

Équipe P.L.H. - E.R.A.S.M.E. (EA4601)

Maison de la recherche (MdR)

5, allées Antonio Machado

F-31058 Toulouse Cedex 9

Tél. : 0033/(0)5.61.50.25.56 et 57

Fax : 0033/(0)5.61.50.24.90 


\section{Historiographie et identités culturelles}

Carole QuATRELIVRe, Le sanctuaire gaulois de Gournay-sur-Aronde (Oise). Retour sur une découverte exceptionnelle des années 1970

\section{Traditions du patrimoine antique}

Christophe CorbIER, Le pindarisme et l'archéologie musicale : style, valeur et authenticité de la première Pythique à l'époque moderne

Arnaud AmiLien, Hélène en Égypte : Hérodote en dialogue avec l'épopée

\section{Archéologie des savoirs}

Sébastien CAzalas, Au jardin des exempla. Rhétorique et stratégie de l'exemplum antique dans l'œuvre politique de Jean Juvénal des Ursins (1388-1473)

Dossier - Perpétuer Ovide : aspects moraux, éditoriaux, linguistiques et culturels $\left(\mathrm{xIV}^{\mathrm{e}}-\mathrm{XvIII}{ }^{\mathrm{e}} \mathrm{s}\right.$.)

Francesca DelL'Oro, Introduction

Hélène Casanova-Robin, L'audace châtiée : Phaéton, Actéon et Icare dans la tradition latine jusqu'à la Renaissance, tours et détours d'un symbolisme

Dylan Bovet, Le commentaire latin des Métamorphoses d'Ovide : pratiques humanistes et évolutions de Regius-Micyllus (1543) à Burmann-Heinsius (I727)

Martine Furno, Ovide en classe, ou un auteur en éclats

Basil Neurs, D'un Ovide chrétien à un Ovide burlesque, du Moyen Âge au Grand Siècle : continuités et changements dans la traduction et dans l'illustration des Métamorphoses perçus à travers deux éditions du xvir siècle

Olivier ThÉvenaz, Épilogue

\section{Actualités et débats}

Tiphaine-Annabelle BESNARD, 2019 : l'année pop des musées d'archéologie. Retour sur l'exposition romaine Il classico si fa pop. Di scavi, copie e altri pasticci

\section{Relire les classiques des sciences de l'Antiquité}

Jean-Pierre AıBERT, Le premier Detienne : une relecture de « La notion mythique d'A $\lambda \hat{\eta} \theta \varepsilon\llcorner\alpha$ » (REG, 1960, p. 27-35)

Marcel DetIENNE, La notion mythique d'A $\lambda \hat{\eta} \theta \varepsilon \varepsilon \alpha$

\section{L'atelier de l'histoire : chantiers historiographiques}

Antiquités numériques (coordonné par Élodie Guillon) (1)

Élodie GuILLON, Introduction

Jaime ALvar, Le projet EPIDI : Epítetos divinos. Experiencia religiosay relaciones de poder en Hispania

Les mots de l'Antiquité (coordonné par Magali Soulatges) (10)

Jack Tномаs, L’Antiquité dans les toponymes de l’État de New York

Actualité du théâtre (coordonné par Malika Bastin-Hammou) (4)

Mathieu Ferrand, “Avons-nous perdu le Soleil ? / Ou l'avons-nous chassé ? ”

Thyeste de Sénèque, traduit par Florence Dupont. Mise en scène de

Thomas Jolly (Avignon, 2018).

Voyages et Voyageurs (coordonné parVéronique Krings) (11)

Claude Azıza, Freud à Pompéi

\section{Comptes rendus de lecture}

